\title{
Chapter
}

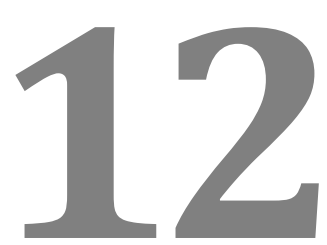

\section{VETERINARY ANTIMICROBIAL STEWARDSHIP}

\section{Hayley Eagar}

South African Animal Health Association (SAAHA), Suite 310, Lagourdia Building, 1262 Embankment Avenue, Centurion 0157, South Africa 


\section{Contents}

12.1. USE OF ANTIMICROBIALS IN VETERINARY MEDICINE AND THE EMERGENCE OF RESISTANCE

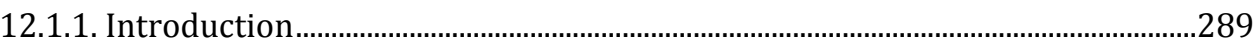

12.1.2. Emergence of resistance in animal health..............................................................290

12.1.3. Current trends of veterinary antimicrobial use in food-producing animals

12.2. MINIMIZING THE RISK OF ANTIMICROBIAL RESISTANCE IN ANIMALS AND

HUMANS - THE WAY FORWARD

12.2.1. World Health Organization (WHO) and other international body initiatives for the containment of antimicrobial resistance in animal health

12.2.2 Other International Regulatory Initiatives to minimise the risk of antimicrobial resistance in animal and human health

12.2.3 Consequences of banning of AGPs and decreasing antimicrobial use in livestock production

12.2.4 Antimicrobial use and resistance surveillance programmes ..............................300

12.2.5 Reducing the need for antimicrobials in animal husbandry ................................302

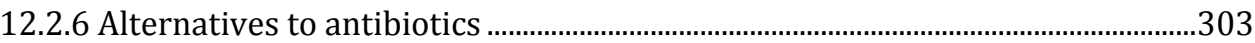

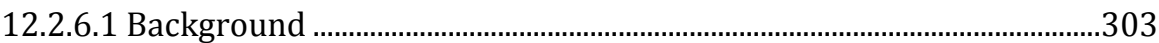

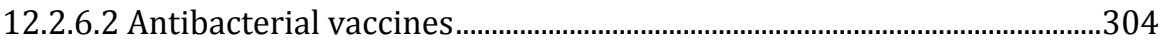

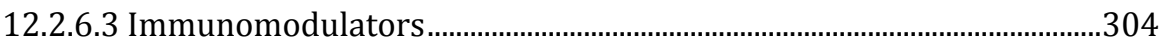

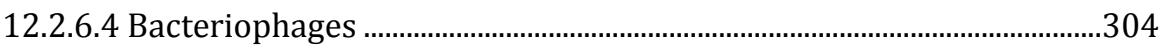

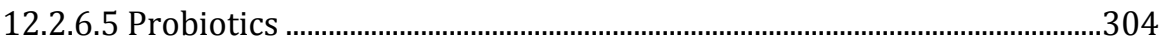

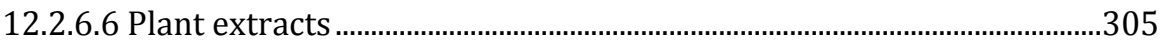

12.2.6.7 Prebiotics.............................................................................................................305

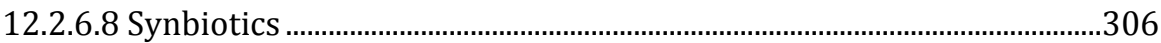

12.2.6.9 Quorum sensing inhibitors .........................................................................306

12.2.7 Other actions to consider and take.............................................................................306

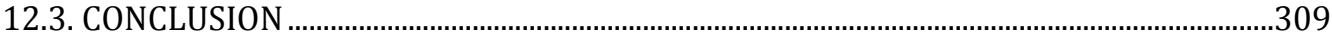

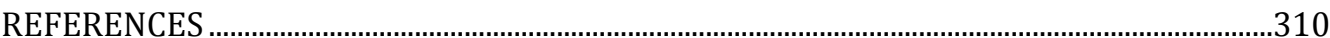




\subsection{USE OF ANTIMICROBIALS IN VETERINARY MEDICINE AND THE EMERGENCE OF RESISTANCE}

\subsubsection{Introduction}

Antimicrobials have played a vital role in human and animal health care for more than 50 years. Antimicrobial resistance (AMR) has escalated rapidly to become a major public health crisis globally. AMR is now a major threat to human and animal health and is placed in the same risk category as climate change and overpopulation. Antimicrobials are important in animal health for the treatment and/or prevention (prophylaxis) of disease in both small companion animals and food animals and also in metaphylaxis and growth promotion in food animals for a sustainable and economically viable animal industry [1]. The various uses of antibiotics in animal health are depicted diagrammatically in Figure 1.

\section{The Uses}
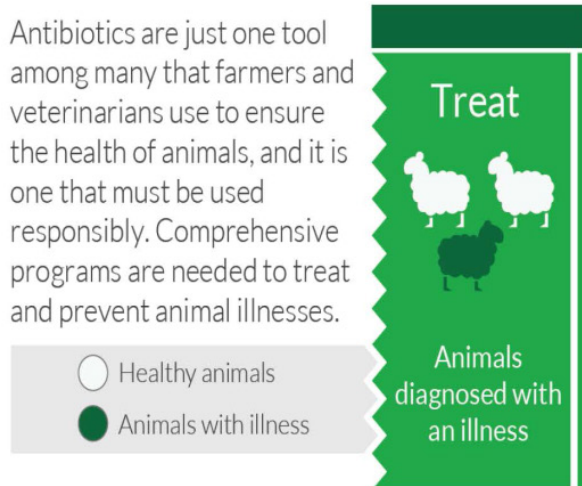

Therapeutic Uses
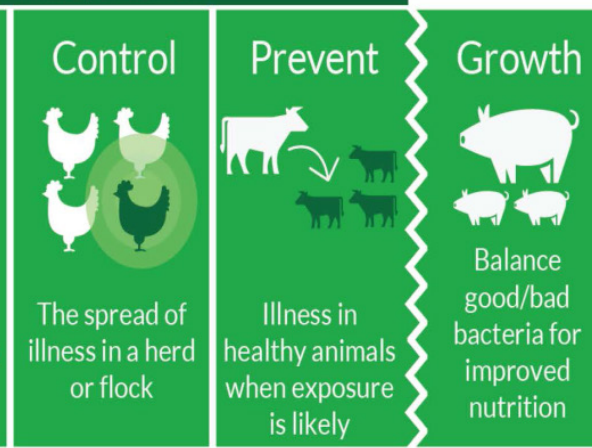

\section{Why Are Antibiotics Used?}

Figure 1. The use of antibiotics in animal health [2] 


\subsubsection{Emergence of resistance in animal health}

However, the overuse and inappropriate use of antimicrobials has resulted in the selection of resistant bacterial populations, as bacteria try to adjust and survive in the unfavourable antimicrobial conditions. Other contributing factors to increasing AMR are:

- Socio-economic disparity with concomitant sanitation issues in many parts of the world

- Poor prescription and dispensing practices

- Lack of national antibiotic resistance surveillance networks

- Inadequate infection control in many hospitals/animal facilities

- The use of antibiotics in feed and water of production animals [2].

Resistant bacteria selected through injudicious use of antimicrobials in companion animals can have a double impact of first making future treatment ineffective as well as having the potential to spread to people through the exchange of resistance genes with bacteria resident in or on the human host. In food animal production, animal bacteria, including resistant strains and/or their resistance determinants may spread to humans by direct contact, through the food chain and by environmental contamination; the two former routes being of major importance [2]. AMR through the food chain may be transferred to humans in the following ways:

- Antibiotic-resistant bacteria pathogenic to humans are selected and the foodstuff becomes contaminated with these bacteria during slaughter and/or the food preparation process. This foodstuff is then ingested and causes an infection for which antibiotic treatment is required and which is ineffective as a result of the antibiotic resistance.

- Antibiotic-resistant bacteria non-pathogenic to humans are selected in the animal and the foodstuff becomes contaminated with these bacteria during slaughter and/or the food preparation process. This foodstuff is then ingested and the bacteria transfer the resistance to other bacteria in the human gut.

- Antibiotics remain as residues in the food animal products which then allows for the selection of antibiotic resistant bacteria in gut of the consumer of this food [1].

Typical zoonotic pathogens include Salmonella spp., Campylobacter spp. and shiga-toxin producing $E$. coli strains. However, many other bacteria are known to spread from animals to humans including other E. coli strains, Yersinia enterocolitica and enterococci [1]. Still, the link between use and overuse of antibiotics and resistance is not easy to follow, as AMR is a very complex and non-victimless phenomenon affecting both human and animal health [3]. 
Figure 2 shows the complex inter-relationship between antibiotic use in humans, animals and the environment.

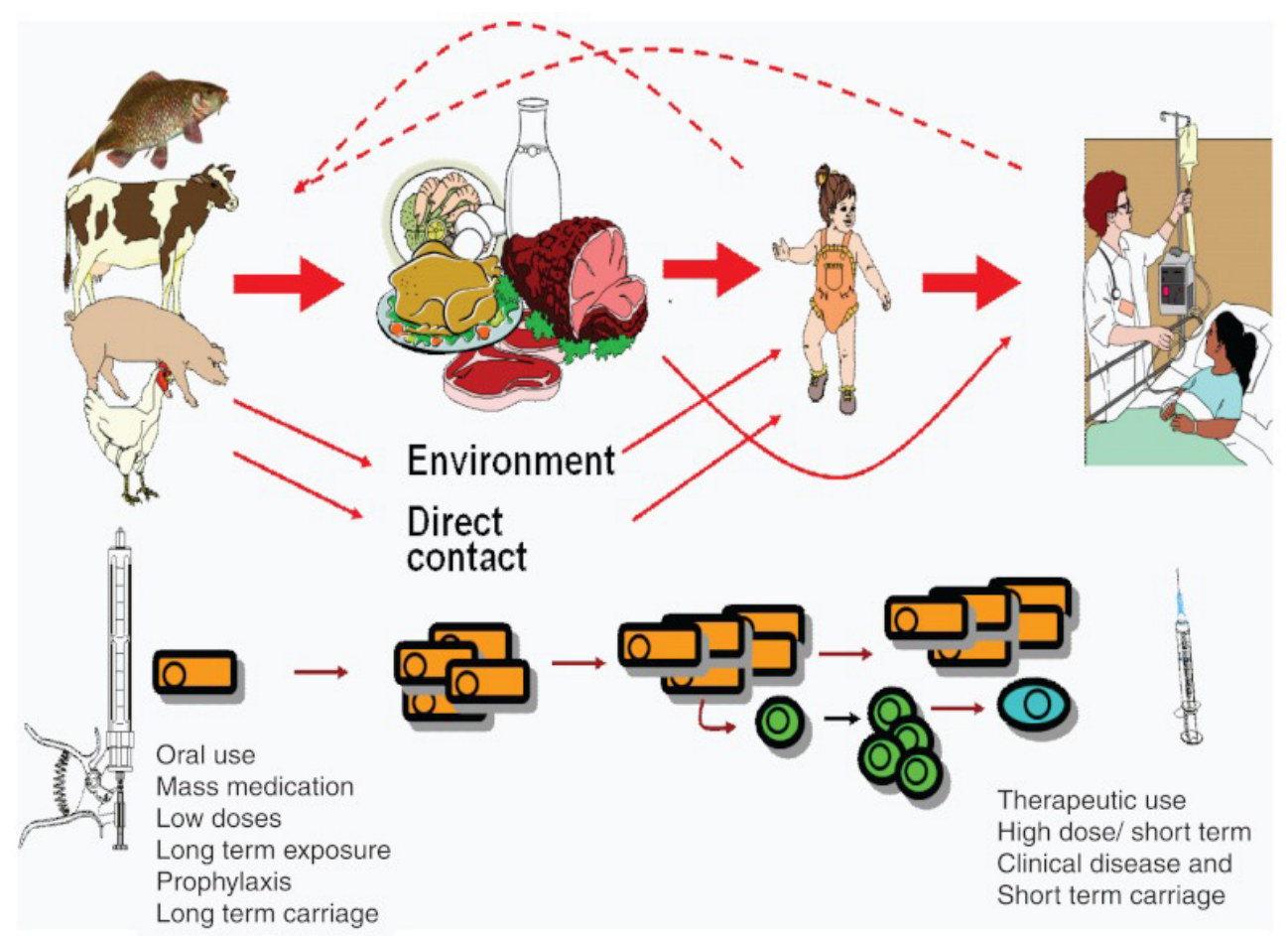

Figure 2. The complex inter-relation between antibiotic use in humans, animals and the environment [4]

\subsubsection{Current trends of veterinary antimicrobial use in food-producing animals}

The use of veterinary antimicrobial agents in food-producing animals in countries of European Union and the United States of America is presented in Table 1 and Table 2, as reported by the European Medicines Agency, and the US Food and Drug Administration (FDA). According to these data, the consumption of antibiotics for animal use has been augmented by about $4 \%$ in the European Union, whereas in the United States, it has followed an ascending trend, despite the call for limiting antimicrobial use in livestock. The FDA however amended the new animal drug regulations in 2015 to implement the veterinary feed directive (VFD) drugs section of the Animal Drug Availability Act of 1996 (ADAA) as from 1 October 2015 which rules the following: A VFD drug is intended for use in animal feeds, and such use of the VFD drug is permitted only under the professional supervision of a licensed veterinarian [3]. 
Table 1. Sales (tons of active ingredient) of veterinary antimicrobial agents applicable mainly for food-producing animals, including horses, on average per European Union member state between 2005 and 2012 [3]

\begin{tabular}{|c|c|c|c|c|c|c|c|}
\hline Antimicrobial class & 2005 & 2006 & 2007 & 2008 & 2009 & 2011 & 2012 \\
\hline Tetracyclines & 148.6 & 143.7 & 157.4 & 134.4 & 119.0 & 123.2 & 113.2 \\
\hline Amphenicols & 1.0 & 1.1 & 1.3 & 1.1 & 1.1 & 2.0 & 2.2 \\
\hline Penicillins & 34.7 & 35.7 & 35.0 & 35.7 & 37.1 & 77.9 & 68.5 \\
\hline Cephalosporins (total) & 1.7 & 1.9 & 1.9 & 1.9 & 1.7 & 0.6 & 0.8 \\
\hline $\begin{array}{l}\text { First- and second-generation } \\
\text { cephalosporins }\end{array}$ & 1.3 & 1.3 & 1.3 & 1.1 & 1.3 & 0.3 & 0.3 \\
\hline $\begin{array}{l}\text { Third- and fourth-generation } \\
\text { cephalosporins }\end{array}$ & 0.4 & 0.4 & 0.4 & 0.4 & 0.4 & 0.6 & 0.5 \\
\hline $\begin{array}{l}\text { Sulfonamides and trimethoprim } \\
\text { (total) }\end{array}$ & 53.7 & 53.3 & 56.6 & 51.6 & 48.7 & 72.6 & 36.7 \\
\hline Sulfonamides & 46.0 & 45.7 & 48.9 & 44.6 & 42.0 & 36.3 & 31.8 \\
\hline Trimethoprim & 7.7 & 7.6 & 7.7 & 7.0 & 6.7 & 5.5 & 5.0 \\
\hline Macrolides & 21.3 & 24.0 & 24.4 & 23.3 & 21.0 & 27.2 & 24.5 \\
\hline Lincosamides & 2.3 & 2.0 & 2.0 & 1.7 & 1.7 & 9.7 & 9.0 \\
\hline Aminoglycosides & 14.6 & 14.4 & 13.9 & 13.0 & 13.0 & 6.6 & 11.2 \\
\hline Quinolones (total) & 4.6 & 4.6 & 4.4 & 4.0 & 3.3 & 10.9 & 7.2 \\
\hline Fluoroquinolones & 0.9 & 1.0 & 1.0 & 0.9 & 1.0 & 5.5 & 5.2 \\
\hline Other quinolones & 3.7 & 3.6 & 3.4 & 2.9 & 2.1 & 2.2 & 1.9 \\
\hline Polymyxins & 10.0 & 10.1 & 11.3 & 10.1 & 10.3 & 22.4 & 21.0 \\
\hline Pleuromutilins & 2.7 & 2.6 & 3.0 & 3.6 & 3.6 & 8.5 & 8.8 \\
\hline Others: & 0.4 & 0.4 & 0.4 & 0.6 & 0.7 & 9.0 & 3.9 \\
\hline Total & 295.6 & 293.7 & 311.6 & 280.9 & 261.1 & 336.8 & 307.0 \\
\hline
\end{tabular}

Notes:

${ }^{*}$ Others include bacitracin, paromycin, spectinomycin, polymyxins, and amphenicols. Data were derived for cumulative reports involving seven European Union member states in 2005-2009, 25 in 2011, and 26 in 2012. Data from the European Medicines Agency. 
Table 2. Antimicrobial drugs approved for use in food-producing animals actively marketed in the United States between 2009 and 2012 (tons of active ingredient) [3]

\begin{tabular}{ccccc}
\hline Drug class & $\mathbf{2 0 0 9}$ & $\mathbf{2 0 1 0}$ & $\mathbf{2 0 1 1}$ & $\mathbf{2 0 1 2}$ \\
\hline Aminoglycosides & 223.12 & 211.79 & 214.89 & 273.53 \\
\hline Cephalosporins & 20.14 & 24.59 & 26.61 & 27.65 \\
\hline Lincosamides & 93.33 & 154.65 & 190.1 & 218.14 \\
\hline Macrolides & 562.06 & 553.23 & 582.84 & 616.27 \\
\hline Penicillins & 691.64 & 884.42 & 885.3 & 965.2 \\
\hline Sulfonamides & 505.9 & 517.13 & 383.1 & 493.51 \\
\hline Tetracyclines & $5,260.99$ & $5,602.28$ & $5,652.85$ & $5,954.36$ \\
\hline Ionophores & $3,739.35$ & 3,820 & $4,122.4$ & $4,573.79$ \\
\hline $\begin{array}{c}\text { Not independently reported } \\
\text { (medically important) }\end{array}$ & 329.39 & 281.22 & 319.99 & 344.43 \\
\hline $\begin{array}{c}\text { Not independently reported } \\
\text { (not medically important) }\end{array}$ & $1,161.54$ & $1,237.78$ & $1,190.94$ & $1,151.53$ \\
\hline Total & $14,618.43$ & $12,587.46$ & $13,287.1$ & $13,569.04$ \\
\hline Notes: & & & &
\end{tabular}

\section{Notes:}

${ }^{*}$ Amphenicols, diaminopyrimidines, fluoroquinolones, and streptogramins;

taminocoumarins, glycolipids, pleuromutilins, polypeptides, and quinoxalines. Data from the FDA Center for Veterinary Medicine.

Abbreviation: FDA, US Food and Drug Administration.

In cattle, antimicrobials such as amoxicillin, penicillin, erythromycin, quinolones, gentamicin, novobiocin, tylosin, tilmicosin, and tetracycline are extensively used. In meat-producing animals, antibiotics are mainly used for the treatment and prevention of bovine pneumonia, diarrhea, and shipping fever, which are the most common problems. For the treatment of pneumonia, oxytetracyclines and spectinomycin are the first-choice antibiotics, with florfenicol and macrolides (particularly tilmicosin) considered as the second choice, with second-, third-, and fourth-generation cephalosporins being the last choice. Still, antibiotics are administered at least once via feed for various reasons, such as liver abscesses, increased growth, and respiratory diseases. The use of narrow-spectrum antimicrobials is favored in cases of clinical mastitis, with first-choice antimicrobials being the $\beta$-lactam antimicrobials used when treating mastitis resulting from streptococci, or penicillin when treating mastitis caused by staphylococci. In certain cases, the use of 
antibiotics intramammary in the non-lactating period is given to the whole herd to prevent infectious mastitis [3].

In pigs, the current trends in husbandry require animal segregation in groups according to age, where pigs are of similar size and weight, and therefore the antimicrobials can be administered in groups of pigs via the oral route by addition in the feed or water. Individual therapy of pigs by injection of antimicrobials is mainly considered in pigs reared for reproduction. Use of antimicrobials for prevention is a common practice in pig farms, especially in stressful periods that predispose for infectious diseases. Such periods are the time between birth and first lactation, where the cut of the umbilical cord and tail and the trimming of the canines takes place; the ablactation period, where the environment and diet change and the castration of males and vaccinations take place; and finally the fattening period, where overcrowding, inadequate aeration, and low or high temperatures can form a quite stressful environment. Prophylactic use of antimicrobials is considered to be higher in the ablactation period, whereas at the end of fattening pigs, they do not receive antimicrobials so as to avoid residues detection after slaughter. For the prevention and treatment of enzootic pneumonia, large quantities of various antibiotics are used, with the most common being ceftiofur, tetracyclines, tiamulin, lincomycin, and enrofloxacin. In addition, in bacterial enteritis, especially when the etiological agent is E. coli or Clostridium perfringens, antibiotic treatment with penicillins, tetracyclines (chlortetracycline, oxytetracycline), quinolones (enrofloxacin), or aminoglycosides (gentamicin, neomycin) is required. Finally, in swine dysentery (Brachyspira hyodysenteriae) and ileitis (Lawsonia intracellularis), lincomycin, tiamulin, macrolides, or tetracyclines are mainly used [3].

In poultry, antibiotics used for therapeutic reasons are usually administered through water, in contrast to growth-promoting use, where antibiotics are added in feed. The most commonly used antibiotics are penicillins (amoxicillin), quinolones (enrofloxacin), tetracyclines (doxycycline, oxytetracycline), macrolides (erythromycin, tylosin), aminoglycosides, the sulfonamide/trimethoprim combination, polymyxins (colistin), and other antimicrobials (tiamulin). In the United States, the above mentioned antibiotics are used, with the exception of fluoroquinolones [3].

The antimicrobials commonly used in sheep and goats are amoxicillin, ampicillin, ceftiofur, the combination of amoxicillin/clavulanic acid, enrofloxacin, erythromycin, lincomycin, oxytetracycline, sulfonamides, penicillin G, trimethoprim and sulfonamide combination, tylosin, and tilmicosin (with the exception of goats, where subcutaneous injection of tilmicosin has been linked to death). Ampicillin, erythromycin, lincomycin, the trimethoprim and sulfonamide combination, and certain sulfonamides (e.g., sulfathiazole) can significantly alter the microbial flora of the rumen when administered per os, and in certain cases, they can lead to death. Therefore, in the mature small ruminants, it is preferable to administer antimicrobials in 
other ways than the oral route (feed or water), with the exception of certain sulfonamides and tetracyclines, which can be absorbed efficiently by the rumen [3].

Concerns about the extensive use of nontherapeutic agents have arisen after the duplication of the antimicrobial use in aquaculture in the decade 1994-2004. In aquaculture animals, several classes of antibiotics have been used. Among them are antibiotics such as sulfonamides, penicillins, quinolones, tetracyclines, and phenicols, which are listed as critically or highly important antimicrobials for human medicine. The last three antimicrobial classes are widely used in salmon farming. Quinolones, tetracyclines, and phenicols are selective for a variety of AMR genes that occur in transposons, plasmids, and integrons that, when mobile, can induce their dissemination [3]. 


\subsection{MINIMIZING THE RISK OF ANTIMICROBIAL RESISTANCE IN ANIMALS AND HUMANS - THE WAY FORWARD}

\subsubsection{World Health Organization (WHO) and other international body initiatives for the containment of antimicrobial resistance in animal health}

- In 1997, the World Health Organisation (WHO) met in Berlin to discuss the medical impact of the use of antimicrobials in food animals (WHO 1997) [1].

- In 2000, the WHO met in Geneva to discuss the global significance of AMR in food animals (WHO 2000) [1].

- In 2003/2004, WHO established the holistic and multi-sectoral approach (One Health) to address the rising threat of AMR. Addressing AMR requires a holistic and multi-sectoral (One Health) approach because antimicrobials used to treat various infectious diseases in animals may be the same or be similar to those used in humans. WHO, the Food and Agriculture Organization (FAO) and the World Organisation for Animal Health (OIE) therefore collaborated to form a tripartite alliance to take collective action to minimize the emergence and spread of AMR [1].

- The first WHO list of CIA was developed in the $1^{\text {st }}$ WHO Expert Meeting on Critically Important Antimicrobials for Human Health held in Canberra, Australia, 2005. During the meeting, participants considered the list of all antimicrobial classes used in human medicine and categorized antimicrobials into three groups of critically important, highly important, and important based on the two criteria developed during the meeting. Table 3 shows the list and classification of antimicrobials that are important in human medicine.

- The Ad hoc Codex Intergovernmental Task Force on Antimicrobial Resistance (TFAMR) was active from 2007-2011 to assess the risks to human health associated with the presence in food and feed including aquaculture and the transmission through food and feed of antimicrobial resistant microorganisms and AMR genes and to develop appropriate risk management advice based on that assessment to reduce such risk [1].

- In 2008, the Advisory Group to the Integrated Surveillance of Antimicrobial Resistance (AGISAR) was established, using the One Health approach [1].

- WHO/Food and Agriculture organisation (FAO)/OIE tripartite alliance's Global Action Plan (GAP) for AMR was published in 2015. All 
member states of the World Health Organisation (WHO) and the Office International des Épizooties (OIE) committed their countries to developing and implementing National Action Plans to control AMR based on the GAP. The GAP strategic objectives are to:

- improve awareness and understanding of AMR through education and training,

- strengthen knowledge and evidence based on surveillance and research,

- reduce the incidence of infection through effective hygiene and infection and prevention control (IPC) measures,

- optimize the use of antimicrobial medicines in human and animal health,

- ensure sustainable investment through research and development [1].

In 2017, the integrated surveillance of AMR in foodborne bacteria using the One Health approach was developed and published with the support of the AGISAR to assist countries and other stakeholders in the establishment and development of programmes of integrated surveillance of AMR in the foodborne bacteria (i.e., bacteria commonly transmitted by food) [5]. There is a schematic representation in Figure 3 of the initiatives undertaken by WHO to address AMR.

\section{Addressing AMR from the Food Chain: Berlin 1997- Geneva 2017 20 years of WHO Commitment}

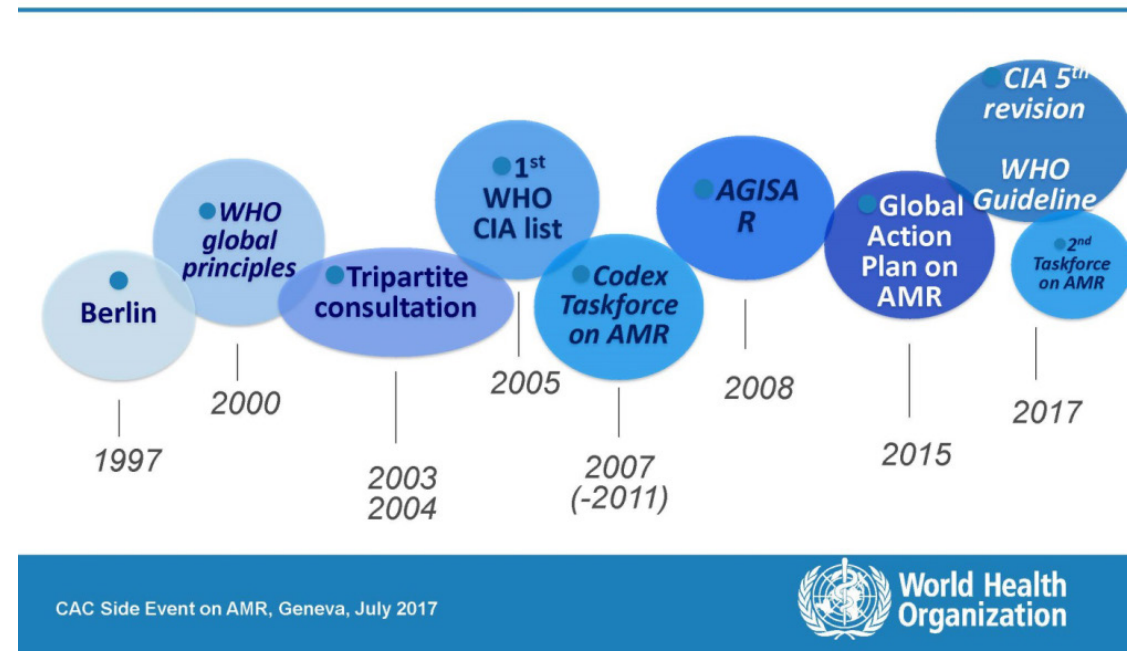

Figure 3. Initiatives by the World Health Organization (WHO) for the containment of AMR [6] 
Table 3. List and classification of antimicrobials important for human medicine [7]

\begin{tabular}{|l|l|}
\hline Antimicrobial class & Example of drug(s) \\
\hline \hline \multicolumn{2}{|l|}{ CRITICALLY IMPORTANT ANTIMICROBIALS } \\
\hline \hline Aminoglycosides & gentamicin \\
\hline Ansamycins & rifampicin \\
\hline Carbapenems and other penems & meropenem \\
\hline $\begin{array}{l}\text { Cephalosporins ( } 3^{\text {rd }}, 4^{\text {th }} \text { and } 5^{\text {th }} \\
\text { generation) }\end{array}$ & ceftriaxone, cefepime, ceftaroline \\
\hline Glycopeptides & vancomycin \\
\hline Glycylcyclines & tigecycline \\
\hline Lipopeptides & daptomycin \\
\hline Macrolides and ketolides & erythromycin, telithromycin \\
\hline Monobactams & aztreonam \\
\hline Oxazolidinones & linezolid \\
\hline $\begin{array}{l}\text { Penicillins (natural, aminopenicillins, } \\
\text { and antipseudomonal) }\end{array}$ & ampicillin \\
\hline Phosphonic acid derivatives & fosfomycin \\
\hline Polymyxins & colistin \\
\hline Quinolones & ciprofloxacin \\
\hline $\begin{array}{l}\text { Drugs used solely to treat tuberculosis } \\
\text { or other mycobacterial diseases }\end{array}$ & isoniazid \\
\hline HIGHLY IMPORT ANT ANTIMICROBIALS \\
\hline Amidinopenicillins & mecillinam \\
\hline Amphenicols & chloramphenicol \\
\hline $\begin{array}{l}\text { Cephalosporins ( } 1^{\text {st }} \text { and } 2^{\text {nd }} \text { generation) } \\
\text { and cephamycins }\end{array}$ & cefazolin \\
\hline Lincosamides & clindamycin \\
\hline Penicillins (anti-staphylococcal) & oxacillin \\
\hline Pseudomonic acids & mupirocin \\
\hline Riminofenazines & clofazimine \\
\hline Steroid antibacterials & fusidic acid \\
\hline Streptogramins & quinupristin/dalfopristin \\
\hline $\begin{array}{l}\text { Sulfonamides, dihydrofolate reductase } \\
\text { inhibitors and combinations }\end{array}$ & sulfamethoxazole, trimethoprim \\
\hline Sulfones & \\
\hline
\end{tabular}

\subsubsection{Other International Regulatory Initiatives to minimise the risk of antimicrobial resistance in animal and human health}

- In the UK it was recommended by the Swann Committee Report (1969) that in legislation, antibiotics must be clearly designated as either "in feed" (i.e. growth promoters) or "therapeutic", and that growth- 
promoting antibiotics should, by definition, have no therapeutic use in humans or animals. This regulation, however, did not include a provision to withdraw approvals of antimicrobial growth promoters (AGPs) should members of the same class at a later time come into use for humans [1].

- From 1986, Sweden was the first of the Scandinavian countries to take the initiative to ban all antimicrobial growth promoters (AGPs). In 1998 Denmark banned AGP use in pig and poultry production [1].

- As a result of the documentation on the use of antimicrobials for growth promotion in food animals leading to the creation of a major food animal reservoir of bacteria resistant to AGPs and also to medically important last resort antimicrobials, such as vancomycin for example, the European Union then imposed a ban on all AGPs that belonged to classes also used in human medicine. Avoparcin was banned in 1997. The EU then also invoked the "Precautionary Principle" in 1998, which propounds that even if there is not enough scientific evidence on the frequency of the risk of a hazard, but nevertheless the hazard is still seen as a possible risk, then this "Precautionary Principle" may be invoked. On this basis, the use of tylosin, spiramycin, virginiamycin and bacitracin were banned as feed additives in EU countries in 1998. Finally, with effect from January $1^{\text {st }}$ 2006, the last four AGPs used in the EU, monensin sodium, salinomycin, flavophospholipol and avilamycin were banned [1].

\subsubsection{Consequences of banning of AGPs and decreasing antimicrobial use in livestock production}

- In Sweden, interestingly enough, the banning of AGPs did not have a detrimental effect on livestock production. For instance, in the production of slaughter pigs, specialized beef, and turkeys, no negative clinical effects were reported as a consequence of the ban. In piglet production, significant clinical problems emerged that created a demand for antibiotic medicated feed at therapeutic dosages. During the subsequent 4-year period, the use of therapeutic antimicrobials increased, involving up to $75 \%$ of the pigs. Thereafter, the use of antimicrobials decreased because of improved management, and was halved in 1993 followed by a gradual further decrease supported by the addition of zinc oxide to the feed. In 1998/1999, only $5 \%$ of weaning piglet producing herds used antimicrobial medicated feed and $17 \%$ used zinc. The AGP ban has shown that under good production conditions it is possible to reach good and competitive production results for the rearing of rearing of poultry, calves, and pigs without the continuous use of AGPs [1]. 
- A systematic review and meta-analysis was done on the effect of interventions to restrict antibiotics in food-producing animals and its associations between antibiotic resistance in food animals and humans. It was concluded that interventions that restrict antibiotic use in foodproducing animals are associated with a reduction in the presence of antibiotic-resistant bacteria in these animals. A smaller body of evidence suggests a similar association in the studied human populations, particularly those with direct exposure to food-producing animals. The implications for the general human population are less clear however, given the low number of studies. These overall findings have directly informed the development of WHO guidelines on the use of antibiotics in food-producing animals [8].

\subsubsection{Antimicrobial use and resistance surveillance programmes}

With the development of resistance being inevitable, there is a need for the regular monitoring of both use trends as well as to ascertain changes in resistance patterns. The epidemiological data gathered can then be used to determine resistance patterns in a specific area in order to provide information that can be included in future treatment protocols. The goal of antimicrobial monitoring and surveillance should therefore be aimed at providing resistance trends that will ultimately be used to evaluate resistance containment interventions. Surveillance programmes need to be adapted to the resources and facilities specific to the various countries and the following are important factors to consider when setting up a surveillance system for antimicrobial use and patterns of resistance [9]:

- Country based - developing countries versus developed countries.

- Monitoring needs to be continuous and long term in order to give an accurate reflection of trends of antimicrobial use and resistance.

- It is essential that the One Health integrated approach is used to triangulate antimicrobial use and the molecular epidemiology of AMR in the food safety bacteria from humans, the food production continuum from farms to retail meat products and associated sewage and water treatment plants.

- There needs to be national harmonisation of sampling, testing methodologies and validations for antimicrobial patterns of resistance.

- Quality Control must also be implemented when establishing and using these surveillance systems.

- Such systems should be flexible as a surveillance programme is a dynamic system that may change as a result of changed circumstances in the country as well as changed patterns of antimicrobial use and resistance. 
- Such surveillance programmes should be driven by the government, whether this is the Ministry of Health or Agriculture and it should be made mandatory for the veterinary pharmaceutical companies to provide the relevant sales statistics of antimicrobials reflected in $\mathrm{kg}$ of active pharmaceutical ingredient (API), according to the class of antimicrobial.

There are surveillance systems in various countries dealing with volumes of veterinary antimicrobials consumed and AMR trends in animals, food, humans and the environment:

- Swedish Veterinary Antimicrobial Resistance Monitoring (SVARM) is a surveillance system for the volumes of veterinary antimicrobials (both food and companion animals) consumed in Sweden as well as AMR trends. This system is part of a holistic approach to limit development of AMR by means of surveillance of AMR and use, control and preventive measures, education, research and training through an agency called the Swedish Strategic Programme for the Rational Use of Antibiotics and Surveillance of Resistance (STRAMA) and is funded by the Swedish government [1].

- Danish Integrated Antimicrobial Resistance Monitoring and Research Programme (DANMAP) was established in Denmark to monitor trends in resistance among bacteria from animals, food and humans, to monitor the consumption of antimicrobial agents and to determine the association between consumption and occurrence of resistance and to model transmission of resistance from animals to humans [1].

- In the United Kingdom, veterinary pharmaceutical companies submit data annually to the Veterinary Medicines Directorate (VMD) on their previous year's sales of antibiotics authorized for use in animals. The VMD use the data provided to calculate the volume of active antibiotic ingredient within the medicines sold. Sales data are used as an estimate for antibiotic usage. However, as not all antibiotics sold will be used, sales figures are generally an overestimate. The VMD collates data from government laboratories on antibiotic resistance in bacteria found in samples from animals. This is managed through two programmes: EU Harmonised Monitoring, which is carried out as a legal requirement, and a clinical surveillance programme, which relies on voluntary submission of samples by farmers and veterinary surgeons. EU Harmonized Monitoring involves the collection of samples from healthy livestock. Samples are tested for the presence of antibiotic resistant bacteria. The bacteria of interest are those which can potentially transfer between animals and man (zoonotic organisms) [1].

- In Australia, trends in the level and type of antimicrobial use are assessed from import records. These records may include information 
on the indications for which the imported antimicrobials will be used. Importers of antimicrobials (merchants, pharmaceutical companies and private individuals) must hold a permit issued by the Therapeutic Goods Administration (TGA) to import antimicrobials. Since 1992, all importers have had to declare the indication of the antimicrobial that they are importing, whether it is intended for human therapeutic use, veterinary therapeutic use, as a growth promoter, research in the laboratory or for another special purpose. The commonwealth Department of Health and Aged Care, through the TGA is responsible for collecting and reporting these end-use data. The TGA issues permits, collects and maintains this information in an electronic system in a tabulated format. The information is expressed as $\mathrm{kg}$ of active ingredient but does not accurately reflect the difference in potencies between agents [1].

- In the United States, surveillance of AMR in food-borne bacteria is undertaken by the National Antimicrobial Resistance Monitoring System (NARMS) for enteric bacteria [1].

\subsubsection{Reducing the need for antimicrobials in animal husbandry}

Treatment of infections with antimicrobials is just one facet of animal husbandry and by adopting the following non-antibiotic best practices animal health may be improved while at the same time the use of antimicrobials is decreased and optimized [2]:

- Improving hygiene practices

- Ensure appropriate sanitation and/or disinfection practices.

- Management

- Consistent access to clean water and dietary adjustments.

- Practice sustainable internal and external parasite control.

- Introduction of prebiotics, probiotics, enzymes, essential oils etc.

- Determine the appropriate stocking density to maintain health and welfare.

- Monitor disease, and maintain accurate and up to date mortality and medication records.

- Introduce specific pathogen free (SPF) flocks or herds.

- Biosecurity

- Ensure biosecurity of operations by isolation/fencing/visitor control.

- Optimize vaccination programmes. 
- Continuously monitor and measure biosecurity and be proactive in improving biosecurity based on these assessments.

- Accountability with use of antimicrobials

- Ensure that there is a responsible, qualified and knowledgeable person in charge of the use of antimicrobials, particularly in intensive operations, such as a veterinarian or other trained person.

\subsubsection{Alternatives to antibiotics}

\subsubsection{Background}

The golden age of the discovery, development and commercialization of new classes of antibiotics and novel antibiotic technologies was during the 1960s and 1970s. However since then research and development of new antibiotics has declined. The reasons for this decreasing interest by the pharmaceutical industry are multifactorial. Bringing a new drug to market requires an average investment of US\$800 million and 10 years or longer. As well, pharmaceutical companies have to support the relatively large research costs of medications that do not make it to market. Furthermore, the risks of post-approval adverse events must also be taken into consideration. Another factor that may play a role in the reduced interest in antibacterial development is the current focus on medications for the treatment of chronic diseases. Unlike medications used to treat chronic diseases, most antibiotic treatments are given for 5 to 14 days and then discontinued. Anti-infectives are intended to quickly eliminate the need for their use. In addition, novel breakthrough antimicrobials often become the agents of last resort, as clinicians and policy makers tend to hold them in reserve, hoping to slow the inevitable emergence of resistance. Also, antibiotics work very well and quickly, and therefore they produce a low return on investment for manufacturers [10].

There have been recent developments in novel technologies that could potentially lead to alternatives to antibiotic use. The feasibility of such alternatives needs to be analysed in depth to ascertain if any of these could possibly substitute veterinary antibiotics in the future. Some of these alternatives include antibacterial vaccines, immunomodulatory agents, bacteriophages and their lysins, antimicrobial peptides (AMPs), pro-, pre-, and synbiotics, plant extracts, inhibitors for bacterial quorum sensing (QS), biofilm inhibitors and plant extracts [11].

Ideal alternatives to antibiotics should: (i) have non-toxic or no side effects on animals, (ii) be easy to eliminate from the body or result in short term residues, (iii) not induce bacterial resistance, (iv) be stable in the feed and animal gastrointestinal tract, ( $\mathrm{v}$ ) be easily decomposed and not affect the environment, (vi) not affect palatability, (vii) not destroy the normal intestinal flora of animals, (viii) kill or inhibit the growth of pathogenic bacteria, (ix) enhance the body's resistance to the disease, $(x)$ improve feed efficiency and 
promote animal growth, and (xi) have good compatibility. In fact, there are no alternatives to antibiotic that currently meet all the above mentioned requirements [11].

\subsubsection{Antibacterial vaccines}

There is still a considerable gap between antibiotic alternatives and antibiotics concerning the effectiveness of disease prevention and growth promotion. Antibacterial vaccines are generally used for the prevention of bacterial infections, and currently only a small number of bacterial infective diseases can be controlled by vaccines. The development of a vaccine that is both practical and inexpensive so that it can be affordable for use in poor countries is still a key problem. The most important challenge for mass immunization with poultry vaccines is the cost of vaccine as well as the feasibility. While vaccines may lessen our reliance on the use of antibiotics, they are complementary rather than a replacement [11].

\subsubsection{Immunomodulators}

Immunomodulators, mainly immunostimulants, are able to non-specifically enhance the innate immune function and to improve the host's resistance to disease. The use of immunotherapy in infectious diseases may resulting in modulating the immune response to a microbe (e.g., by using cytokines and cytokine inhibitors), modifying a specific antigen-based response (e.g., using interferons) and minimizing end-organ damage using non-specific antiinflammatory agents (e.g., steroids). Immunomodulators and feed enzymes mainly preserve the health of animals, but do not directly kill or inhibit bacteria [11].

\subsubsection{Bacteriophages}

Bacteriophages are viruses that are parasitic on bacteria. Bacteriophages are currently only used in food, and the safety is still questionable [11].

\subsubsection{Probiotics}

Probiotics have been defined by the WHO as "microorganisms which, administered live and in adequate amounts, confer a benefit to the health of the host." Probiotics can kill and prevent pathogenic microorganisms by producing antimicrobial compounds such as bacteriocins and organic acids, improve gastrointestinal microbial environment by adherence to intestinal mucosa thereby preventing attachment of pathogens and competing with pathogens for nutrients, stimulate the intestinal immune responses and improve the digestion and absorption of nutrients [11]. There is a diagrammatic representation of the action of probiotics in the animal intestinal tract in Figure 4. 


\subsubsection{Plant extracts}

Plant materials are used widely in traditional systems of medicine. Plant extracts, also known as phytobiotics, have been exploited in animal nutrition, particularly for their antimicrobial, anti-inflammatory, anti-oxidative, and antiparasitic activities. However, the composition of plant extracts and probiotics is complex and the quality in terms of stability is poor, resulting in varying effects and safety risks [11].

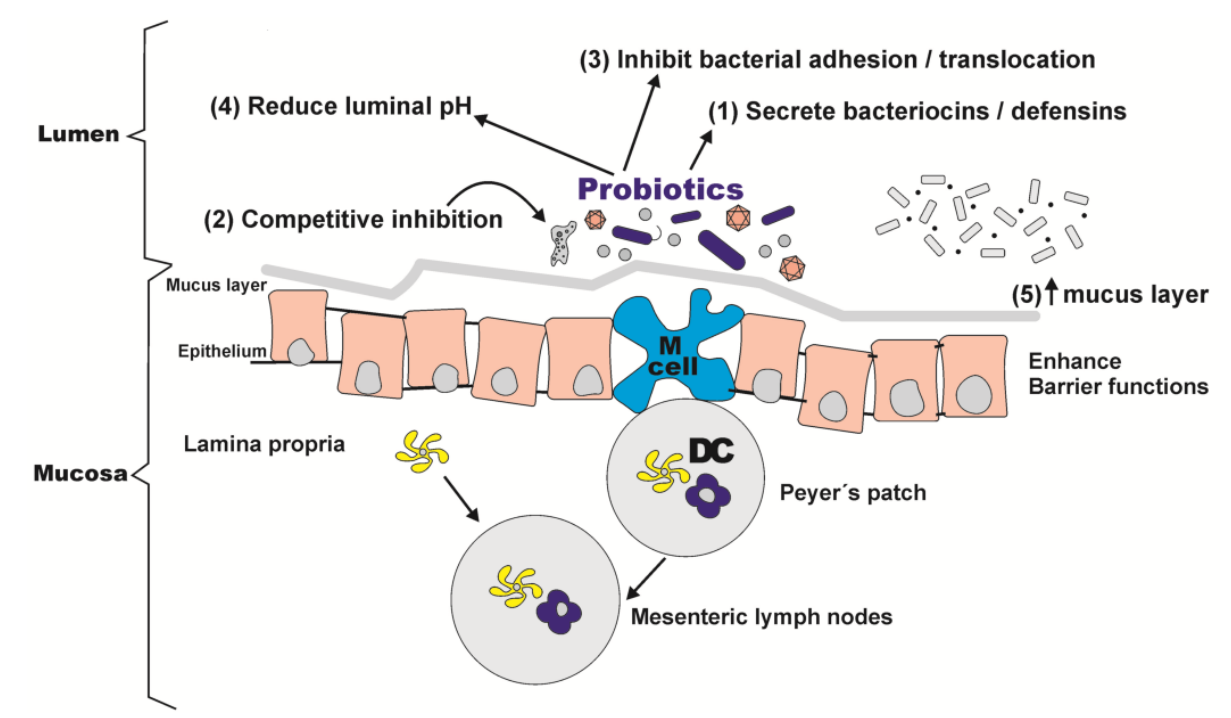

Figure 4. Mechanism of action of probiotics within the intestinal tract of animals [12]

\subsubsection{Prebiotics}

Prebiotics are non-digestible (by the host) food ingredients that may have a beneficial effect through their selective metabolism in the intestinal tract. Prebiotics include oligosaccharides, polysaccharides, natural plant extracts, protein hydrolysates, polyols, etc. Prebiotics are stable compounds with no residues, no induced resistance, and may be supplied from a wide variety of sources. However, many prebiotic products are not authorized currently in the EU as feed additives under the commission regulation (EC) 1831/2003. This is due to certain drawbacks to these products. Firstly, prebiotics themselves cannot inhibit and kill pathogens, thus they cannot prevent or treat bacterial infections as antibiotics do. Secondly, feeding a large quantity of prebiotics may cause bloating, diarrhea, and other adverse reactions in livestock due to the fermentation in the gastrointestinal tract [11]. 


\subsubsection{Synbiotics}

Synbiotics are the joint preparations of probiotics and prebiotics, and therefore have their dual role. There are some reports on the beneficial effects of synbiotics on the physiological and production indices of piglets such as the enhancement of production performance in piglets and the improvement of average daily gain. However, the data from these reports on the beneficial effects of synbiotics in swine production are still limited [11].

\subsubsection{Quorum sensing inhibitors}

Bacterial pathogenicity is, in part, under the regulation and control of the QS system. A QS system consists of self-induced signaling molecules (autoinducers [AIs]), receptors, and downstream regulatory proteins. Inhibitors targeting the QS system can block its functions and thereby inhibit the bacterial pathogenic effects controlled by the QS system. Inhibitors targeting QS and pathogenicity of bacteria are still being researched with no approved products, and most QSIs are toxic to eukaryotic cells. Biofilms are structured consortiums of bacteria embedded in a self-produced polymer matrix consisting of polysaccharide, protein and DNA. Biofilm-forming bacteria may cause chronic infections because they show increased tolerance to antibiotics and disinfectants as well as resisting phagocytosis and other defense mechanisms of the body. Biofilm inhibitors show good results only when used in combination with antibiotics. AMPs include products such as gramicidin, polymyxin, bacitracin, and sugar-peptide. Although AMPs can treat bacterial infections, the high cost and narrow antibacterial spectrum restrict their wide use, and they can still induce bacterial resistance [11].

Antibiotics can directly inhibit or kill bacteria with better antibacterial effect than all the antibiotic alternatives mentioned here. Moreover, antibiotics are formulated from relatively pure active ingredients and the quality is ensured by good manufacturing practice. So far, no antibiotic alternative has satisfactorily replaced the clinical efficacy of appropriate antibiotics used in bacterial infections [11].

\subsubsection{Other actions to consider and take}

The question to be asked is whether any antimicrobial use can select for resistance i.e. sub-therapeutic levels over an extended period in the feed for growth promotion versus high doses? The World Veterinary Association (WVA) has recommended 'use as long as needed but for the shortest duration possible [2].

Other actions to take for an integrated approach to veterinary antimicrobial stewardship are: 
- Prudent use guidelines to be adopted or adapted from international bodies such as the OIE that are applicable and practical to all the various animal species in the various countries [2].

- The Veterinary International Conference of Harmonisation (VICH) is a trilateral (EU-Japan-USA) programme aimed at harmonising technical requirements for veterinary product registration. The VICH GL27 is the Guidance on Pre-Approval Information for Registration of New Veterinary Medicinal Products for Food Producing Animals with Respect To AMR is one of the VICH guidelines. It is recommended that pre-registration requirements for antimicrobials be in accordance with this VICH guideline. Furthermore, the Regulatory Authorities (RAs) also play a pivotal role in AMR by determining local registration requirements, availability of antimicrobials on the market as well as access to antimicrobials (for example, control by veterinarians). RAs may determine that local Minimum Inhibitory Concentration (MIC) data be collated for a representative sample of the bacteria for which claims are made, withdrawal periods to be set in food animals, flagging and evaluation of any potential antimicrobials for resistance that may impact on human health, and pharmacokinetic/pharmacodynamic (PK/PD) relationships of antimicrobials to optimize the use of concentration dependent bactericidal versus time dependent bactericidal antimicrobials in order to maximize safety and efficacy but minimize the risk of selection for resistant bacterial populations [13].

- Some countries have a dual registration system of over the counter (OTC) antimicrobials versus veterinary prescription only which leads to inconsistencies in registration requirements. Such systems need to be evaluated with the view to having one regulatory agency to control antimicrobials or harmonised registration requirements for both OTC and scheduled veterinary antimicrobials [13].

- Do not use critically important and highly important antimicrobials to human medicine, except for treatment and when there is no alternative [14].

- Ensure that no compounded medicines are used in food animals or extra label use of medicines, except as a last line resort. If antimicrobials are compounded as a last resort for food animals, then Good Manufacturing Practice (GMP) or Good Pharmacy Practice (GPP) need to be implemented during the production to ensure the highest quality medicine possible. A suitable withdrawal period also needs to be established by the veterinarian to ensure that the consumer is not exposed to any antimicrobial residues that may lead to AMR [14].

- The Pharmaceutical Inspection Co-operation Scheme (PIC/S) leads the international development, implementation and maintenance of harmonised GMP standards and quality systems of Inspectorates in the 
field of medicinal products. It is important for countries to be part of such systems in order to standardise the quality of medicines globally [15].

- There need to be incentives for the research and development of novel antibiotics, such as research grants [10].

- Use pharmacovigilance as a tool to also establish AMR trends as a lack of efficacy of an antimicrobial is also classified as an adverse drug reaction (ADR).

- The Regulatory Authorities need to be very vigilant on preventing counterfeit medications from reaching the market as such medicines may not have the correct API, or the incorrect quantity of API or may be of sub-standard quality amongst other issues that may contribute to the AMR problem.

- The undergraduate and postgraduate curriculum of all animal science, medical science and veterinary students needs to introduce more information on the importance of rational antimicrobial use to minimize the development of AMR and the interrelationships between AMR in humans, animals and food products and the environment $[1,13]$.

- The efficacy of traditional antibiotics can still be improved. Some "old" antibiotics can find new bacterial targets and be used against some multi-drug resistant (MDR) bacteria. It has been demonstrated that in many cases, there are non-carbapenem alternatives for the treatment of extended-spectrum- $\beta$-lactamase-producing $E$. coli (ESBL-Ec) infections. Novel formulations can also allow targeted drug delivery via nanoparticles to reinforce the antimicrobial effect of these antibiotics. Furthermore, in empirical therapy, use of broad-spectrum bactericidal agents that will eradicate the presumed infective microorganism(s), which potentially could be MDR, should be selected. Once an infection is under control and the culture and susceptibility results are reported, it is important to then switch to the most suitable narrow-spectrum antibiotic thereby decreasing the potential of adverse drug effects and the risk of development of antibiotic-induced resistance [11]. 


\subsection{CONCLUSION}

We must not forget that "prevention is better than cure". However, for many developing countries, because of the poor farming environment and the high incidence of disease, antibiotics are still an effective tool in the prevention and control of animal diseases. It was proven that the ban of growth promoters in EU resulted in a requirement for the improvement of the farm hygiene. After the amount of "old" antibiotics used in feed was reduced due to the ban, the prevalence of bacterial infections in the target animals was shown to increase if there was no fundamental improvement of hygiene and animal husbandry in the production environment. This may lead to increased therapeutic use of antibiotics and result in some unintended consequences that would be cause for new public health concerns. The benefit versus risk should be assessed before implementing such policies as a result of political/social pressure, as bacteria may not necessarily "listen" to the policy-makers. Thus, the decision concerning the use of in-feed antibiotics should be made based on scientific approaches. The ban of antibiotics as growth promoters cannot be implemented in every country of the world [11].

Again, the integrated One Health approach to antimicrobial stewardship and resistance needs to be emphasized. All stakeholders, from the various governmental agencies, veterinary pharmaceutical industry, veterinarians, para-veterinarians, agricultural sector, food processors, abattoirs, retail shops, animal scientists, medical profession, companion animal owners and the public need to work together as a cohesive whole, in order to resolve this crisis [13]. Antibiotics must be used responsibly and there must be continuous development of alternatives to antibiotics to ensure the long-term sustainability of the existing antibiotics. Antimicrobial use with respect to target animals, duration of the treatment, withdrawal period, prudent use as well as regulations/policies must be strictly defined. At the same time, the supervision and enforcement of such laws must be strengthened in order to control antibiotic resistance. It is essential that antimicrobial use and resistance surveillance programmes are implemented that are applicable, sustainable and practical to the socioeconomic development and agricultural policies of the various countries. Furthermore, we must improve the management of animal nutrition and production hygiene, since recent European developments showed a distinctly more positive outcome from the ban of antibiotic growth promoters than was anticipated due to the improvement of animal welfare.

It is absolutely essential that the awareness of AMR is now translated into the action of veterinary antimicrobial stewardship in order to preserve the efficacy of our antimicrobials for our future generations to also enjoy the good healthcare that they have a right to receive! 


\section{REFERENCES}

1. H. Eagar, G. Swan, M. Van Vuuren, A survey of antimicrobial usage in animals in South Africa with specific reference to food animals, Dissertation in support of MMED (Veterinary Pharmacology) at the University of Pretoria, 2008.

2. M. van Vuuren, Inception Workshop of World Health Organization (WHO) Advisory Group to the Integrated Surveillance of Antimicrobial Resistance (AGISAR), Managing the global challenge of antibiotic resistance in animal husbandry - perspectives from the Ministerial Advisory Committee (MAC), University of Kwazulu-Natal, College of Health Sciences, South Africa, 2017.

3. V. Economou, P. Gousia, Infect. Drug Resist. 8 (2015) 49-61.

4. Institute of Medicine (US) , Improving Food Safety Through a One Health Approach: Workshop Summary. Washington (DC): National Academies Press (US); 2012. Available from: https://www.ncbi.nlm.nih.gov/books/NBK100665/ doi: 10.17226/13423. (accessed 3 February 2018).

5. $\quad$ S. Essack, Inception Workshop of World Health Organization (WHO) Advisory Group to the Integrated Surveillance of Antimicrobial Resistance (AGISAR), The 'One Health' Approach to Containing Antimicrobial Resistance, University of Kwazulu-Natal, College of Health Sciences, South Africa, 2017.

6. A. Aidara-Kane, Inception Workshop of World Health Organization (WHO) Advisory Group to the Integrated Surveillance of Antimicrobial Resistance (AGISAR), Leading and Shaping Global Work on Antimicrobial Resistance from the Food Chain Using a "One Health" Approach. 20 Years of WHO commitment, University of Kwazulu-Natal, College of Health Sciences, South Africa, 2017.

7. Critically important antimicrobials for human medicine $-5^{\text {th }}$ rev. Geneva: World Health Organization; 2017. Licence: CC BY-NC-SA 3.0 IGO.

8. $\quad$ K.L.Tang, N.P. Caffrey, D.B. Nobrega, Lancet Planet. Health 1 (2017) e316e327.

9. P.J. Fedorka-Cray, Inception Workshop of World Health Organization (WHO) Advisory Group to the Integrated Surveillance of Antimicrobial Resistance (AGISAR), Antimicrobial Resistance Surveillance Systems: The Global Experience, University of Kwazulu-Natal, College of Health Sciences, South Africa, 2017.

10. J.M. Conly, B.L. Johnston, Can. J. Infect. Dis. Med. Microbiol. 16(3) (2005) 159160.

11. G. Cheng, H. Hao, S. Xie, X. Wang, M. Dai, L. Huang, Z.Yuan, Front. Microbiol. 5 (2014) 217.

12. L.K. Otutumi, M.B. Góis, E.R. de Moraes Garcia, M.M. Loddi, Veterinary Medicines and Science, Probiotic in Animals, Chapter 9, Variations on the Efficacy of Probiotics in Poultry, InTech, open access, 2012.

13. H. Eagar, V. Naidoo, Biol. Rev. 1(2) (2017), http://journals.kei.org/index.php/ibr/article/view/1367 (accessed 23 November 2017).

14. South African Veterinary Council (SAVC), Professor Moritz van Vuuren, Director: Food Safety and Security Portfolio of the SAVC, Pretoria, South Africa 2017.

15. Pharmaceutical Inspection Co-operation Scheme (PIC/S), online at: https://www.picscheme.org/. Website accessed February 2018. 
(C)2018 by the authors; licensee IAPC, Zagreb, Croatia. This chapter is an open-access publication distributed under the terms and conditions of the Creative Commons Attribution license (http://creativecommons.org/licenses/by/3.0/) (cc) BY 
